\title{
Los primos del riesgo y las variaciones del índice general de la bolsa de valores de Lima (IGVBL)
}

\author{
"MANERAS DE CÓMO SE CONSTRUYEN LOS RESULTADOS \\ DEL MERCADO".
}

Econ. Juan Alberto BACIGALUPO POZO

\section{RESUMEN}

El presente documento elaborado, más que un artículo o u ensayo; es una curiosidad, la cual investigo hace más de siete años y que obedece una serie de análisis previos, que comprenden, el buscar nuevas maneras que permitan interpretar la información de los mercados más grandes que tienen mucha incidencia en los resultados de los mercados más pequeños. Presento un esquema de interpretación, descomponiendo los resultados del IGBVL en factores primos para responder a las interrogantes planteadas. Profundizar aún más este análisis, significará elaborar otro documento similar. Espero que estas líneas sean de su agrado.

\section{ABSTRACT}

Prepared herein, more than one item test ou; It is a curiosity, which I investigate more than seven years and reflects a number of previous analyzes, which include the search for new ways to enable interpret the information in the larger markets that have much impact on the results of the smaller markets. I present a scheme of interpretation, the results of the General Index breaking into prime factors to answer the questions raised. Further deepen this analysis, develop other similar document meant. I hope these lines are to your liking.

Podemos argumentar y adjudicar una serie de acontecimientos en los mercados de capital internacionales que hacen sentir sus efectos en la bolsa de valores de Lima; mercado pequeño que no representa ni el $0.0005 \%$ de lo que se negocia en el mundo en un día; y estoy siendo generoso con este dato. Las bolsas de Nueva York, México y Brasil, son mil veces más grandes en montos negociados. Solo esta distancia en términos de montos nos revela que, cualquier movimiento 
en los mercados más grandes, por más pequeños que estos sean, afectan significativamente el rendimiento de nuestra bolsa, podemos decir o citar, que: el grado de dependencia nos afecta; o que las empresas más grandes del Perú negocian en el NYSE (New York Stock Exchange) y también esto nos afecta; o que el riesgo es más sistemático que otra cosa, etc., podemos seguir formulando, inventando y repitiendo una serie de adjetivos, para entender la influencia de otros mercados en las variaciones del IGBVL (Índice General de la Bolsa de Valores de Lima). Estos detalles, nos indican dos cosas que parecen atrevidas: primero, que los métodos y las técnicas de análisis de los riesgos ya no son suficientes y segundo, que estamos empleando mal algún método o técnica que no nos permite entender por qué nuestro mercado se ve afectado.

O tal vez que, esta complicación se origina en nuestro mercado y las soluciones también.

En 1905, Albert Einstein, en un artículo sobre mecánica estadística manifestaba que, "La desviación estándar del desplazamiento de una partícula suspendida en un líquido en un tiempo dado, es proporcional a la raíz cuadrada de dicho tiempo", si interpretamos esto, es igual a $\operatorname{Ln}\left(\frac{V F I G B V L}{V I I G B V L}\right) \boldsymbol{x} V \mathrm{n}$ y calculamos la desviación estándar del mercado en un determinado momento; en finanzas e inversiones, desviación estándar es sinónimo de riesgo.

Actualmente para tratar de entender los riesgos y la influencia de otros mercados en los rendimientos de las bolsas; el análisis se realiza en tiempo continuo y en ambientes estocásticos, podemos citar el cálculo de Ito (conocido también como la integral de Ito), donde se estudian variables aleatorias cuya dinámica es guiada por ecuaciones diferenciales, las cuales tienen un componente estocástico, que toma como base, la teoría del movimiento browniano, o el modelo Black F., Scholes M . (1973) que se utiliza en la valuación de derivados financieros, el mismo que toma en cuenta en su análisis el modelo CAPM (Modelo de precios de los activos de capital). Podemos coincidir que todo análisis de los resultados y riesgos están ligados a un modelo o enfoque matemático, que derivan en una gran variedad de formas de analizar los mercados en un contexto dinámico, continuo y estocástico. Asimismo las contribuciones de la física en el análisis a través de los fractales, el efecto mariposa, la percolación, entre otros, nos aproximan más a la noción de que los riesgos que afectan nuestro mercado y a sus resultados, ocurren por sucesos que se dan simultáneamente originados por las expectativas de los inversionistas que incorporan toda la información de todos los mercados en un plazo muy breve; del mismo modo, cada suceso simultáneo tiene múltiples soluciones.

Pero ¿Cómo puedo determinar la cantidad de sucesos simultáneos que inciden en las variaciones del IGBVL ya que estas variaciones incorporan 
expectativas cuando se interpreta la información de los mercados?; ¿Cómo puedo asociar la información de forma tal que al observar el desempeño del mercado y sus resultados independientemente a que estos sean positivos o negativos se pueda conocer de cuantas maneras se construyó este resultado en un ambiente continuo y dinámico?

Para conocer tal situación y aproximarnos a la cantidad de sucesos, tenemos que recurrir a los primos del riesgo. Incorporamos a este análisis a los números primos. Pero ¿Qué es un número primo? Son los números enteros positivos que solo son divisibles por sí mismos y por uno; el 3 y el 5, son ejemplos de números primos. Los números compuestos, se escriben como producto de varios primos. En conclusión cuentan con propiedades matemáticas especiales por ser los más pequeños, todos los demás números se componen a partir de estos; son como lo genes del cuerpo humano o los átomos que componen la materia. (Los 23 pares de cromosomas, las 3 hélices básicas del ADN son números primos).

Dicho esto y asumiendo que cuando se incorpora la información del mercado y esta se considera como un todo, los números primos se consideran como las variaciones más pequeñas de los mercados más grandes que afectan el desempeño y los resultados del IGBVL. Al tomar una muestra pequeña del IGBVL, del 2 al 30 de setiembre del 2014, el resultado en puntos del índice lo descomponemos en factores primos y esto nos ayuda a determinar la cantidad de sucesos simultáneos que producen los mercados más grandes y que tienen un impacto en nuestro mercado. El resultado del índice en términos absolutos, siempre es un número compuesto de muchos primos y este a su vez nos explica de cuantas maneras se construyó el resultado en el IGBVL. Tal como podemos observar en el siguiente cuadro:

Cuadro $\mathrm{N}^{\circ}$ 1: Al descomponer los resultados en factores primos y responder a las interrogantes planteadas, debemos de considerar lo siguiente: Los datos más grandes representan la cantidad de sucesos simultáneos que se producen en el mercado por expectativas cuando se interpreta la información. Los datos más pequeños representan la cantidad de mercados con mayor información por interpretar. Los datos intermedios representan, las reacciones que se generan cuando los mercados con mayor información no terminan de interpretarla. 


\begin{tabular}{|c|c|c|}
\hline & RESULTADO EN PUNTOS & DESCOMPOSICON DEL RESULTADO \\
\hline FECHA & IGBVL & IGBVL EN NUMEROS PRIMOS \\
\hline 02/09/2014 & 17014 & $2 \times 47 \times 181$ \\
\hline 03/09/2014 & 17219 & $67 \times 257$ \\
\hline 04/09/2014 & $17041(* x)$ & \\
\hline 05/09/2014 & 17229 & $3 \times 5743$ \\
\hline 08/09/2014 & 17249 & $47 \times 367$ \\
\hline 09/09/2014 & 17139 & $3 \times 29 \times 197$ \\
\hline $10 / 09 / 2014$ & 16989 & $3 \times 7 \times 809$ \\
\hline $11 / 09 / 2014$ & $17077(* *)$ & \\
\hline $12 / 09 / 2014$ & 16926 & $2 \times 3 \times 7 \times 13 \times 31$ \\
\hline $15 / 09 / 2014$ & 17012 & $2 \times 2 \times 4253$ \\
\hline $16 / 09 / 2014$ & 17075 & $5 \times 5 \times 683$ \\
\hline $17 / 09 / 2014$ & 17002 & $2 \times 8501$ \\
\hline $18 / 09 / 2014$ & 16909 & $37 \times 457$ \\
\hline $19 / 09 / 2014$ & 16942 & $2 \times 43 \times 197$ \\
\hline $22 / 09 / 2014$ & 16769 & $41 \times 409$ \\
\hline $23 / 09 / 2014$ & 16788 & $2 \times 2 \times 3 \times 1399$ \\
\hline $24 / 09 / 2014$ & 16725 & $3 \times 5 \times 5 \times 223$ \\
\hline $25 / 09 / 2014$ & 16621 & $11 \times 1511$ \\
\hline $26 / 09 / 2014$ & 16601 & $13 \times 1277$ \\
\hline $29 / 09 / 2014$ & 16568 & $2 \times 2 \times 2 \times 19 \times 109$ \\
\hline $30 / 09 / 2014$ & 16227 & $3 \times 3 \times 3 \times 601$ \\
\hline
\end{tabular}

Fuente: Bolsa de valores de Lima, resultados del índice en cifras absolutas del mes de setiembre.

Para entender mejor la construcción de los resultados analicemos que pasó el 29 de setiembre y como se construyó el resultado de 16568 puntos del IGBVL con una variación negativa de $(-0.20 \%)$.

Los índices de los mercados más grandes, registraron un débil desempeño, afectados por las expectativas sobre cuando la reserva federal americana (FED) incrementaría su tasa de referencia así como las tensiones en medio oriente. Los índices más importantes del mundo cerraron con cifras negativas, el S\&P 500 (-009\%); DOW JONES (-0.30\%) y el NASDAQ (-0.03\%), el resultado de los mismos en la última semana también fue negativo en $(-1.40 \%),(-1.00 \%)$ y $(-1.50 \%)$, respectivamente. (Son los mercados con mayor información por procesar).

Las ventas de viviendas existentes en el mes de agosto fueron menores de lo esperado, las ventas de nuevas viviendas aumentaron de manera significativa durante el mismo periodo, las órdenes de bienes duraderos cayeron drásticamente a (-18.20\%), el índice de confianzas calculado por la Universidad de Michigan retrocedió levemente, el índice anualizado del PBI del segundo trimestre se mantuvo en línea con lo proyectado por el consenso y las solicitudes de seguros de desempleo se ubicaron ligeramente por debajo de lo esperado, situación que ayudó a que el mercado reaccionara de forma positiva. 
Cuadro $\mathrm{N}^{\circ}$ 2: Muestra los índices de estados Unidos, de los commodities y de las principales bolsas latinoamericanas al cierre del 29 de setiembre del 2014.

\begin{tabular}{|c|c|c|c|c|c|}
\hline \multicolumn{2}{|c|}{$\begin{array}{l}\text { PRINCIPALES INDICES USA } \\
\text { AL CIERRE 29.09.14 }\end{array}$} & \multicolumn{2}{|c|}{$\begin{array}{c}\text { COMMODITIES } \\
\text { AL CIERRE 29.09.14 } \\
\end{array}$} & \multicolumn{2}{|c|}{$\begin{array}{c}\text { BOLSAS LATINOAMERICANAS } \\
\text { AL CIERRE } 29.09 .14 \\
\end{array}$} \\
\hline S \& P 500 & $-0.09 \%$ & ORO & $0.24 \%$ & IPC (MEX) & $-0.05 \%$ \\
\hline DOW JONES & $-0.30 \%$ & PLATA & $0.02 \%$ & BOVESPA & $-4.36 \%$ \\
\hline \multirow[t]{3}{*}{ NASDAQ } & $-0.03 \%$ & COBRE & $0.43 \%$ & IPSA (CHI) & $-0.62 \%$ \\
\hline & & PETROLE WTI & $1.11 \%$ & COLCAP & $-0.28 \%$ \\
\hline & & PETROLEO BRENT & $0.22 \%$ & IGBVL & $-0.20 \%$ \\
\hline
\end{tabular}

Fuente: Bolsa de Valores de Lima, resultados bursátiles del 29 de setiembre de 2014.

Las variaciones de los principales commodities, las bolsas latinoamericanas y las principales bolsas de Europa y Asia que procesan más de 4,500 noticias por día, son los datos intermedios que representan la reacción de los mercados cuando los que cuentan con mayor información no han terminado de interpretarla.

Los demás datos (el primo más alto), representan los sucesos simultáneos que se producen por las expectativas que afectaron a todos los mercados y el desempeño del IGBVL en ese día, de tal forma que también se construye el resultado del índice, el cual queda determinado por: Los mercados con mayor información por procesar; los datos de los mercados cuando la información no se procesa en su totalidad y los sucesos simultáneos por expectativas.

$$
\text { Resultado del IGBVL }=2^{3} \text { X } 19 \text { X } 109=16568
$$

Resultado que también nos indica, de cuantas formas reaccionan los mercados a la vez, al interpretar la información simultánea en tiempos muy cortos (15 segundos, 1, 5, 10, 30 minutos o una hora), etc. De esta forma podemos hacerlo con cualquier otro día.

Juzguen ustedes mismos, ya que esto no deja de ser una novedosa forma de analizar los resultados de los mercados más grandes que impactan en mercados muchos más pequeños.

\section{BIBLIOGRAFÍA}

1. Álvarez González, Alfonso "Análisis bursátil con fines especulativos". México D.F. Editorial Limusa, 2005.

2. Atehortua Granados, John Alexander "Mercado de Capitales y portafolios de inversión”. Medellín, Ediciones de la U. 2012.

3. Ayala Brito, Gamaliel "Finanzas Bursátiles". Editorial México D.F. Instituto Mexicano de contadores públicos. 201. 
4. Ton Soo, Tang "Matemáticas aplicadas a los negocios, las ciencias sociales y de la vida”. México D.F.: Cengage Learning. 2012.

5. Venegas Martínez, Francisco "Riesgos Financieros y Económicos: Productos Derivados y Decisiones Económicas bajo incertidumbre”. México D.F.: Cengage Learning. 2008. 